\title{
PUNTUALIZACIONES SOBRE LA RELACIÓN DE SIMULTANEIDAD EN EL SISTEMA TEMPORAL DEL VERBO ESPAÑOL
}

\begin{abstract}
Resumen. Las caracterizaciones aspectuales propuestas en la última gramática académica para los valores expresados por las distintas formas verbales evidencian claros paralelismos con propiedades temporales. Las defensas de un valor aspectual imperfectivo en el caso de aquellas formas cuyas realizaciones temporales básicas incluyen alguna relación de simultaneidad («perfecto compuesto», «presente», «imperfecto») tropiezan antes o después con empleos de dichas formas no caracterizables en términos aspectuales de imperfectividad, lo que de nuevo pone en evidencia las perturbaciones derivadas de la aplicación a la descripción gramatical de una lengua románica como es el español de conceptos trasvasados del análisis de otros tipos de lenguas.
\end{abstract}

Palabras clave: verbo español, vector de simultaneidad, aspecto imperfectivo.

1. Partiendo del análisis de las que consideramos realizaciones temporales básicas de las nueve funciones temporales identificables en el sistema verbal español, y representándolas mediante las formulaciones vectoriales en su día propuestas por Rojo (1974; cfr. también Rojo \& Veiga, 1999: § 44.2.2.3), observamos la aparición de, en concreto, un vector de simultaneidad $(\mathrm{oV})$ tanto en las correspondientes a las que a la manera bellista llamamos relaciones de presente, $\mathrm{OoV}$, y co-pretérito, $(\mathrm{O}-\mathrm{V}) \mathrm{oV}$, expresadas respectivamente por canto y cantaba en Indicativo 0, como también en aquella a que el maestro venezolano se refirió como antepresente, $(\mathrm{OoV})-\mathrm{V}$, la representada por he cantado en dichas circunstancias modales no marcadas.

Como ya hemos puntualizado anteriormente (cfr. Veiga, 2013: $\S 6$ ), entre las caracterizaciones de signo aspectual propuestas en la última gramática académica y el análisis vectorial de Rojo se advierten claros paralelismos. En concreto, la NGLE atribuye la consideración de «imperfectivas» exclusivamente a las antedichas formas en cuyas realizaciones temporales básicas interviene un vector de simultaneidad,

\footnotetext{
* Universidade de Santiago de Compostela, Campus de Lugo.
} 
mientras que por otro lado califica de «neutras» aquellas que expresan alguna realización básica de posterioridad primaria (vector $+\mathrm{V}$ a la derecha) $\mathrm{y}$ de «perfectivas» las que expresan anterioridad primaria (vector $-\mathrm{V}$ a la derecha), olvidando curiosamente a habría cantado:

\begin{tabular}{|l|l|l|}
\hline \multicolumn{1}{|c|}{ Forma verbal (IND0) } & $\begin{array}{c}\text { Rojo (1974), } \\
\text { Rojo \& Veiga (1999) }\end{array}$ & $\begin{array}{c}\text { NGLE (§ 23.2k) } \\
\text { (caracterizaciones aspectuales) }\end{array}$ \\
\hline canto & $\mathrm{OoV}$ & forma imperfectiva \\
\hline cantaba & $(\mathrm{O}-\mathrm{V}) \mathrm{oV}$ & $"$ \\
\hline cantaré & $\mathrm{O}+\mathrm{V}$ & forma neutra en cuanto al aspecto \\
\hline cantaría & $(\mathrm{O}-\mathrm{V})+\mathrm{V}$ & $"$ \\
\hline he cantado & $(\mathrm{OoV})-\mathrm{V}$ & forma imperfectiva o perfectiva \\
\hline canté & $\mathrm{O}-\mathrm{V}$ & forma perfectiva \\
\hline había cantado & $(\mathrm{O}-\mathrm{V})-\mathrm{V}$ & $"$ \\
\hline habré cantado & $(\mathrm{O}+\mathrm{V})-\mathrm{V})$ & \multicolumn{2}{c|}{} \\
\hline habría cantado & $((\mathrm{O}-\mathrm{V})+\mathrm{V})-\mathrm{V})$ & \\
\hline
\end{tabular}

Estos paralelismos descriptivos constituyen nuevas evidencias de la imposibilidad de señalar en el sistema verbal español diferencias funcionalmente aspectuales identificables partiendo de una identidad de contenidos temporales, hecho en el que siempre ha descansado nuestro rechazo a la admisión del aspecto como categoría gramatical independiente en la estructura de dicho sistema (cfr., p. ej., Veiga, 1992: $\S 5$; cfr. también Rojo \& Veiga, 1999: § 44.4). Y si pasamos a atender, en concreto, a las caracterizaciones aspectuales defendidas en la NGLE en el caso de las formas indicativas que expresan alguna realización temporal básica que incluya una orientación de simultaneidad, observaremos que dicha gramática señala para canto, cantaba y he cantado la posibilidad compartida de expresar la variedad «continua» del aspecto imperfectivo, que «se caracteriza por focalizar cierta situación incluida en un determinado intervalo» (NGLE: § 23.2o), descripción fácilmente reinterpretable en términos temporales como manifestación de un enfoque de simultaneidad, ya que, respecto del punto de referencia desde el que se enfoca directamente el proceso, cualquier situación que haya comenzado en un momento anterior y todavía esté desarrollándose puede orientarse en tal perspectiva.

No puede dejar, por otra parte, de llamar nuestra atención la dispar caracterización terminológica en lo referente a las tres formas verbales de indicativo aspectualmente no clasificadas en la NGLE ni como imperfectivas ni como perfectivas. Si al lado de uno y otro valores aspectuales se parte del reconocimiento de la doble posibilidad tanto en 
los casos de cantaré y cantaría como en el de he cantado, queda sin explicar por qué las dos primeras aparecen descritas como aspectualmente «neutras» (cfr. supra) pero no se ha aplicado idéntica caracterización a la última forma a la que, por ejemplo, Thibault (2000: 213, 224) se había referido precisamente como un «tiempo neutro»-paralelo en este aspecto al «presente»-, o, invirtiendo los términos, por qué si en este último caso se nos habla de una «forma imperfectiva y perfectiva», no se ha empleado la misma doble caracterización aspectual para cantaré ni para cantaría. Una vez más se nos hace patente la identificación implícita entre tales propiedades aspectuales y las orientaciones identificables en las señaladas realizaciones temporales básicas correspondientes a unos y otros valores gramaticales, tal como más arriba hemos indicado; por tal motivo la NGLE atribuye la aludida doble caracterización a la forma verbal transmisora de un contenido temporal en cuya manifestación básica reconocemos, como ya Bello (1841: § 69) cuando analizaba su «antepresente», la presencia de una orientación (la primaria) de anterioridad al lado de una segunda orientación, esta de simultaneidad («coexistencia» para Bello).

2. El coherente reconocimiento de esta doble composicionalidad temporal en que interviene la simultaneidad originaria evita, ya para empezar, las paradójicas atribuciones, sobre la base de su posible empleo para procesos iniciados en el pasado y aún no concluidos ( $c f r$., p. ej., la noción de "pretérito perfecto continuo» en NGLE: § 23.7ñ), de «imperfectividad» a una forma tradicionalmente llamada "pretérito perfecto» ${ }^{1}$. Al respecto, no descubrimos nada nuevo si, con Azpiazu y Kempas (2017: § 2), argumentamos que la tipología de los usos de dicha forma que ha sido trasvasada al estudio del verbo español desde lo que Comrie (1976: § 3.1 y ss.) escribió partiendo de los hechos gramaticales del inglés puede reducirse a dos casos principales, en términos de «continuativo»-el verbo refiere una acción que persiste en el momento de la enunciación- frente a «no continuativo»-el verbo refiere una acción concluida en algún momento pasado-, respectivamente ejemplificados en (1a) y (1b):

(1a) Siempre me ha gustado la música [= me sigue gustando]

(1b) La conferencia se ha celebrado esta misma mañana [= no se sigue celebrando].

${ }^{1}$ Elocuentemente se planteó Cartagena (2001), por ejemplo, que «resulta difícil justificar el ser del no ser, a saber, la perfectividad imperfectiva», y Moreno de Alba (2006: n. 31, cfr. 2002: 82) escribiría: «Convengo en que no suena nada elegante hablar de perfectos imperfectivos», para concluir que «en la lengua española el tiempo llamado pretérito perfecto (he cantado) en ocasiones no es aspectualmente perfectivo». 
Como tampoco es la primera vez que exponemos (cfr., p. ej., Veiga, 2013: $\S 11$, el reconocimiento de un componente temporal de simultaneidad originaria en el significado gramatical del «pretérito perfecto» español explica sin problemas que un proceso primariamente enfocado como anterior a una referencia simultánea al origen pueda no haber concluido todavía en el momento de su configuración temporal lingüística, pues basta con que resulte anterior a algún punto inserto en la duración total de dicha referencia, que, como gramaticalmente "presente», puede abarcar una parte de lo que cronológicamente es «futuro». En cuanto a las apariciones de he cantado refiriendo hechos o procesos ya concluidos, es en la presencia de dicha orientación originaria de simultaneidad donde, en confrontación con la perspectiva gramatical propia del valor expresado por canté, radican las tan reiteradas observaciones sobre su capacidad para comunicar hechos pasados de alguna manera vinculados a algo que es «presente» (Veiga, 2013: $§ 12$ ).

3. En lo que respecta a las formas de «presente», bien a la vista está la combinabilidad de su valor gramatical con diferentes dimensiones aspectuales, considerando, para empezar, el significado léxico del verbo y sus proyecciones en usos lingüísticos particulares, que pueden ir de lo estrictamente «puntual» a lo más «general»:

(2a) Muere al caer desde un sexto piso

(2b) Su hijo estudia Filología

(2c) Dos y dos son cuatro.

Un ejemplo como (2a), que ilustra un empleo bien característico de titular de prensa en español, revela la aplicabilidad de un enfoque temporal «presente» a un hecho aspectualmente puntual sin que forzosamente se obtenga como resultado una manifestación de iteratividad o habitualidad. Esta última proyección aspectual, aun esperable y, si se quiere, la más espontánea en las circunstancias comunicativas más comunes -en que el centro deíctico de relaciones temporales se sitúa en el «ahora» en que tiene lugar la comunicación- no es ni mucho menos exclusiva de verbos de Aktionsart «puntual»; ejemplos como (3a-b) o, ya no digamos, (3c) así nos permiten observarlo sin mayores problemas:

(3a) Siempre me levanto a las siete

(3b) Desayuno café con leche

(3c) Últimamente duermo muy mal².

2 No podría, de hecho, hablarse de estricta «puntualidad» en los dos primeros casos, en los que se alude a procesos cuyo tiempo de desarrollo habilita su enfoque gramatical mediante, por ejemplo, una perífrasis aspectual progresiva como es estar + gerundio; $c f r$ : 
No obstante, (2a) nos ofrece una manifestación aspectualmente semelfactiva y en modo alguno imperfectiva del hecho verbalmente expresado que entronca con el bien conocido caso del "presente histórico», resultante del reenfoque temporal de unos hechos que, acaecidos en un momento cronológicamente pasado, hallarían su más espontánea configuración gramatical por medio de ciertos valores integrados por una relación de anterioridad, como, para empezar, los expresados por un «pretérito» (ej. 4a), para pasar a ser enfocados en una perspectiva de simultaneidad a un centro de referencias retrospectivamente desplazado, que abandona así su coincidencia «por defecto» con el momento del acto verbal para permitir la expresión de los mismos hechos por parte de una forma de «presente» (ej. $4 \mathrm{~b})$ :

(4a) No te lo vas a creer: ayer me lo encontré por la calle y el muy tonto fue y me dijo...

(4b) No te lo vas a creer: ayer me lo encuentro por la calle y el muy tonto va y me dice...

En este caso, como fácilmente se observa, la aplicación de la mencionada retrotracción del centro deíctico de orientaciones temporales -posibilidad, como sabemos, no exclusiva de las formas de «presente» (cfr., p. ej., Veiga, 1987: § 2.4; Rojo \& Veiga, 1999: § 44.2.2.5)-, provoca frente a su mencionada situación "por defecto» una alteración en el enfoque temporal del proceso verbal, pero ningún cambio gramatical propiamente descriptible en términos aspectuales. Los «presentes históricos» constituyen, por tal motivo, fuente propicia de ejemplos de formas verbales de «presente» transmisoras de contenidos gramaticales no calificables de imperfectivos, como es lógico que suceda cuando la forma en cuestión resulta de la reorientación retrospectiva del enfoque que, con respecto a la ubicación más habitual de dicho centro deíctico, viene a ser el de directa anterioridad primaria, $\mathrm{O}-\mathrm{V}$, expresado en el Indicativo 0 castellano por la forma canté.

A un análisis aspectual en términos de forzosa perfectividad llevará igualmente la observación de ciertos empleos de las formas de «presente»

(3a') Ya se está levantando, pero tendrás que esperar a que se haya duchado

$\left(3 \mathrm{~b}^{\prime}\right)$ Están desayunando en el piso de arriba

$\left(3 c^{\prime}\right)$ No hagas ruido, que los niños ya están durmiendo,

perífrasis cuyo valor aspectual no excluye, por otro lado, la transmisión de un contenido de iteración o habitualidad en los contextos oportunos; cfr.:

(3a") Últimamente me estoy levantando a las siete

$\left(3 b^{\prime \prime}\right)$ Últimamente estoy desayunando café con leche

$\left(3 c^{\prime \prime}\right)$ Últimamente estoy durmiendo muy mal.

De hecho, puede afirmarse en términos generales que esta perífrasis, como igualmente cualquier forma verbal, puede hacer contextualmente referencia tanto a acciones o procesos singulares como a sucesiones iterativas. 
en actos «performativos» en que la mismísima pronunciación de la forma supone el cumplimiento de lo significado por el verbo correspondiente; cfr.:

(5a) Se abre la votación

(5b) Se levanta la sesión

(5c) Os declaro marido y mujer.

Para una forma verbal que admite estos usos, obvia debe resultar la inadecuación de cualquier caracterización aspectual en términos de imperfectividad, que conduciría a la absurda interpretación de que en (5a) la votación no hubiera llegado a abrirse del todo ni en (5b) la sesión a levantarse, o de que en (5c) el matrimonio continuase sin ser establecido. Huelgan, creemos, mayores comentarios.

4. En cuanto a la forma verbal tradicionalmente llamada «pretérito imperfecto», ya hemos reflexionado (p. ej., en Veiga, 2008: § 11.6.2) acerca de la imposibilidad de señalar un valor aspectual imperfectivo ante ejemplos del tipo

(6a) Observaron horrorizados que la bomba hacía explosión dos minutos antes de lo previsto

(6b) Transcurrido el tiempo reglamentario, el partido finalizaba con el resultado inicial

(6c) En 1824, en el pueblecito austriaco de Ansfelden, nacía Anton Bruckner

y poco procedería añadir ahora al respecto. Sí queremos subrayar que la noción de imperfectividad se caracteriza, por definición -ya en la misma etimología del término-, por la ausencia de un límite temporal para el desarrollo de un proceso, sentido en que ha sido definida o admitida por los defensores del aspecto en la descripción gramatical del verbo español. Así, por ejemplo, la NGLE (§ 23.2k) nos dice del «pretérito imperfecto», que, como forma imperfectiva, "presenta la acción en su curso, sin referencia a su inicio o su fin», y, más recientemente, para Bravo (2017: $\S 4.2)$ «el aspecto Imperfecto por definición no permite ver los límites». Tal definición prototípica no facilita la admisión de cualquier valor aspectual no terminativo en usos bien característicos de la forma verbal cantaba como aquellos en que refiere una situación finalizada en el pasado que, como tal, se desea contraponer a una realidad vigente en el presente, $c f r$.:

(7a) En mis tiempos de la escuela había mucho respeto hacia los maestros [= ya no lo hay]

(7b) ¡Pero con lo bien que se comía antes en este restaurante! [= ya no se come]

(7c) ¡Con lo educada que era esta niña de pequeñita! [= ya no lo es].

Y, desde luego, debiera excluir la construcción de cualquier forma funcionalmente imperfectiva acompañada de marcadores gramaticales que señalen precisamente un límite final para el proceso expresado por 
el verbo, lo que no viene a ser en modo alguno el caso del «pretérito imperfecto» español, perfectamente combinable con este tipo de marcadores tal y como muestran los ejemplos del tipo:

(8a) Aquí se comía muy bien hasta que empezó la guerra

(8b) Nos lo estábamos pasando muy bien hasta que llegó el pesado de tu cuñado

(8c) Ni siquiera sabía quién era yo hasta que hace un par de años... (NGLE: § 23.13g).

No obstante, lenguas con el aspecto gramaticalizado como categoría funcional en sus sistemas verbales, y pensamos en el conocido caso eslavo, admiten la conjugación imperfectiva en construcciones correspondientes a nuestros anteriores ejemplos (W. Nowikow, comunicación personal), mas tampoco de esta posibilidad habríamos de extraer conclusiones precipitadas sabiendo, como sabemos, que los hechos aspectuales señalados en los sistemas eslavos no guardan relación con la oposición gramatical que las lenguas romances establecen entre parejas de formas del tipo canté / cantaba (cfr., p. ej., Veiga, 2008: § 11.6).

5 . Seguimos, en cualquier caso, y ante los hechos de un sistema de corte románico en que no puede probarse la independencia funcional del aspecto respecto de la categoría temporal, siendo reticentes ante toda definición en términos de «imperfectividad» del valor gramatical de unas formas que admiten empleos no compatibles con tal definición. La descripción gramatical del español sigue viéndose perturbada por el recurso al trasvase de conceptos únicamente verificables como funcionales en las estructuras de otras lenguas, como, en este caso, la noción de aspecto en el propio núcleo del sistema verbal (con el griego clásico y las lenguas eslavas actuando desde un principio como referentes) con resultados como las señaladas caracterizaciones inapropiadas, cuando no decididamente contradictorias con los hechos, o bien las desviaciones descriptivas de nociones que se defienden como aspectuales pero son presentadas en términos genuinamente temporales (cfr., p. ej., Veiga, 2008: § 11.4.1). En el caso concreto en que nos hemos detenido, el reconocimiento de los efectos varios de una relación temporal de simultaneidad en combinación con diversas realizaciones de aspecto léxico o diferentes configuraciones aspectuales de un predicado conduce a la adecuada caracterización de ciertos valores gramaticales integrados en el sistema verbal español que han sido objeto de discutibles descripciones desde un punto de vista aspectual. 


\section{Bibliografía}

AZPIAZU, S. y KEMPAS, I. (2017). «Acerca del uso prehodiernal del pretérito perfecto compuesto resultativo en español», Zeitschrift für romanische Philologie, 133/3, 709-727.

BELLO, A. (1841). Análisis ideolójica de los tiempos de la conjugacion castellana. Valparaíso: Imprenta de M. Rivadeneyra. Repr. facs. Caracas: Cromotip, 1972.

BRAVO, A. (2017). «Problemas para una definición del aspecto prospectivo», Moenia, 23, 419-445.

CARTAGENA, N. (2001). «Conservación y variación como factores de divergencia del verbo español en América. Posibilidades y límites de convergencias normativas», II Congreso Internacional de la Lengua Española. «El Español en la Sociedad de la Información». Valladolid [en línea] <http://www.congresosdelalengua.es/valladolid/ponencias/unidad_diversidad_del_espanol/2_el_espanol_de_america/cartagena_n.htm>, fecha de consulta: 29 de abril de 2019 .

COMRIE, B. (1976). Aspect. An Introduction to the Study of verbal Aspect and Related Problems. Cambridge: Cambridge University Press, reimpr. 1989.

MORENO DE ALBA, J. G. (2006). «Valores verbales de los tiempos pasados de indicativo y su evolución», en C. COMPANY COMPANY (ed.), Sintaxis histórica del español. Primera parte: La frase verbal. México D. F.: U.N.A.M. / Fondo de Cultura Económica, vol. 1, 5-92.

NGLE - Real Academia Española \& Asociación de Academias de la Lengua Española (2009). Nueva gramática de la lengua española. Morfología. Sintaxis I. Madrid: Espasa Libros.

ROJO, G. (1974). «La temporalidad verbal en español», Verba, 1, 68-149.

ROJO, G. y VEIGA, A. (1999). «El tiempo verbal. Las formas simples», en I. BOSQUE y V. DEMONTE (dirs.), Gramática descriptiva de la lengua española, vol. 2. Madrid: Real Academia Española / Fundación José Ortega y Gasset / Editorial Espasa Calpe, 2867-2934.

THIB AULT, A. (2000). Perfecto simple y perfecto compuesto en español preclásico. Tübingen: Max Niemeyer.

VEIGA, A. (1987). «El presente histórico como hecho de sistema verbal», Verba, 14, 169-216. Reed. en Veiga (2002: 15-56).

VEIGA, A. (1992). «La no independencia funcional del aspecto en el sistema verbal español», Español Actual, 57, 65-80. Reed. en Veiga (2002: 103-117).

VEIGA, A. (2002). Estudios de morfosintaxis verbal española. Lugo: Tris-Tram.

VEIGA, A. (2008). «Co-pretérito» e «irreal» / «imperfecto»o «inactual». El doble valor gramatical de cantaba en el sistema verbal español y algunos problemas conexos. Lugo: Axac.

VEIGA, A. (2013). «La anterioridad del 'pretérito perfecto'». Conferencia pronunciada en el simposio Formas simples y compuestas del pasado en español y otras lenguas. Universidad de Salamanca, 18-20.09.2013, en S. AZPIAZU (ed.), Formas simples y compuestas de pasado en el verbo español. Lugo: Axac, 2014, 147-177. 